INVESTIGACIÓN / RESEARCH

Recibido: 30/03/2015 Aceptado: 08/06/2015 Publicado: 15/07/2015

\title{
EL TRATAMIENTO INFORMATIVO DE ANA BOTELLA DURANTE SU MANDATO COMO ALCALDESA DE MADRID
}

Beatriz Catalina-García1: Universidad Rey Juan Carlos. España. beatriz.catalina@urjc.es

José Gabriel Fernández-Fernández Universidad Rey Juan Carlos. España. josegabriel.fernandez@urjc.es

\section{RESUMEN:}

La figura de Ana Botella como alcaldesa de Madrid ha generado cierta controversia, principalmente en acontecimientos de gran trascendencia pública, algunos de ellos de extrema gravedad. Desde su nombramiento directo y sin pasar por las urnas (noviembre del 2011) hasta su renuncia a presentarse para la siguiente legislatura (diciembre 2014), se han sucedido otros tres hechos de extraordinaria importancia mediática en los que la alcaldesa y el ayuntamiento han desempeñado un papel protagonista. En este trabajo, con apenas precedentes en la literatura académica sobre el ámbito político municipal, se analiza el tratamiento informativo que la prensa nacional de Madrid ha realizado en torno a la alcaldesa. Como principal objetivo se observa la valoración positiva, negativa o neutra hacia Ana Botella y si su presencia en los medios ha sido relevante. En total se han extraído y analizado 1.419 piezas informativas o de opinión incluidas en las ediciones madrileñas de los diarios $A B C$, El Mundo, El País y La Razón. Entre las conclusiones más significativas destaca el escaso protagonismo mediático de la alcaldesa en los acontecimientos estudiados, a pesar de la implicación directa en ellos del Ayuntamiento que preside.

PALABRAS CLAVE: Ana Botella - alcaldesa - medios de comunicación administración municipal - impacto mediático - periódicos

\section{THE INFORMATIVE TREATMENT OF ANA BOTELLA DURING HIS TENURE AS MAYORESS OF MADRID}

\section{ABSTRACT:}

${ }^{1}$ Beatriz Catalina-García: Doctora en Ciencias de la Comunicación (2011) y Licenciada en Ciencias Políticas (2014). Profesora visitante de la Universidad Rey Juan Carlos (Madrid), ha publicado diversos 
The figure of Ana Botella as Madrid mayoress has generated some controversy mainly in events of great public importance, some of them of utmost gravity. Since its direct appointment bypassing the polls (November 2011) until he resigned to stand for the next elections (December 2014), there have been three other facts of extraordinary media importance in which the mayoress and the City Council have played a role protagonist. In this work, with little precedent in the academic literature about the municipal political scope, is analyzed the coverage that the national press of Madrid has done about the mayoress. Is observed as main objective the positive, negative or neutral valuation to Ana Botella and if their presence in the media has been relevant. In total were extracted and analyzed 1,419 news items or opinion contained in the Madrid edition of ABC, El Mundo, El Pais and La Razon daily. Among the most significant findings, stand out the lack of media attention a the mayoress in the events studied, despite direct involvement the City Council presiding.

KEY WORDS: Ana Botella - mayoress - mass media - local government - media impact - newspappers

\section{INTRODUCCIÓN}

Desde el nombramiento como alcaldesa de Madrid en noviembre del 2011, incluso antes, en su cargo como concejala de su predecesor en el cargo Alberto RuizGallardón, la figura de Ana Botella ha sido motivo de polémica desde dos frentes básicos, uno de ellos vinculado al régimen democrático español y el otro relacionado con factores externos. En el primer caso ya generó controversia al ser llamada a encabezar el gobierno municipal sin haber pasado por las urnas y, en segundo lugar, su ascenso en la política ha suscitado dudas sobre si fue consecuencia de sus méritos propios o por su relación matrimonial con el que fuera presidente del gobierno español, José María Aznar.

Aparte de estas apreciaciones, en apenas 33 meses de gestión, Botella se ha debido enfrentar a diversas situaciones cuanto menos incómodas y en ocasiones de extrema gravedad, como el suceso ocurrido en noviembre de 2012 de la avalancha humana en el recinto Madrid Arena provocada por serios problemas de organización que tuvieron como resultado la muerte de cinco jóvenes. El inicio en el cargo ya no fue fácil, la crisis económica ha afectado profundamente a la gran mayoría de los gobiernos locales $y$, de forma notable, al de Madrid que arrastraba un endeudamiento cifrado en más de 7.100 euros, el mayor de todos los ayuntamientos de España.

Diversas encuestas han reflejado, en líneas generales, una importante valoración negativa de su mandato. Como ejemplo de ellas, El País, el diario con mayor tirada en España, entrevistó telefónicamente a 600 madrileños en mayo de 2013, de los 
cuales, el $77 \%$ calificaba con un suspenso su trabajo al frente del ayuntamiento madrileño, el más bajo recibido con respecto a los otros tres líderes de los grupos políticos que conformaban la corporación: Jaime Lissavetszky por el PSOE, Ángel Pérez de IU y David Ortega de UPyD. En la misma encuesta un $53 \%$ consideraba que su gestión era peor que la desarrollada por su antecesor y mentor, Alberto RuizGallardón, aunque en este último dato hay que reseñar que un $83 \%$ de los que contestaron negativamente a esta pregunta confirmaron que en las últimas elecciones municipales (2011) votaron a partidos distintos al que milita Ana Botella (Partido Popular).

Cuatro meses después de su renuncia, en septiembre de 2014, el diario La Vanguardia registró, también a través de una encuesta, que el $85 \%$ de los encuestados consideraba muy malo su mandato al frente del Ayuntamiento madrileño, el 5 por ciento lo calificó como malo, y únicamente el 10 por ciento restante se situaba en una franja valorativa entre aceptable a muy bueno.

\subsection{Marco teórico}

Si bien la literatura científica ha sido profusa en analizar, desde diversos puntos de vista, la relación entre política y los medios de comunicación tradicionales; en el plano investigador y durante los últimos años apenas se han registrado referencias que ofrezcan la visión periodística de un líder en particular. Las existentes se basan fundamentalmente en jefes de Estado (Domínguez, 2012; Váldez \& Rivera, 2009; Grebe, 2007; Castillo, 2003), mientras que se relega a un plano absolutamente secundario el protagonismo mediático que puedan tener los representantes municipales.

Desde Horton y Wohl (1956), que vieron la forma de empatizar de los votantes y los líderes políticos a través de los medios, los procesos electorales en los diferentes ámbitos geográficos han servido también para analizar la influencia de los medios de comunicación. Aira (2009:34) asegura que uno de los factores básicos para el éxito en una campaña electoral de un líder político es su aparición en el máximo número posible de espacios de televisión, incluso en aquellos que son ajenos a los servicios informativos. En este sentido, pone como ejemplo, aparte de cameos en series de entretenimiento, el uso que hizo en 2005 el entonces presidente de gobierno, José Luis Rodríguez Zapatero para divulgar en el reality-show de Gran Hermano el Tratado de la Constitución Europea.

Sin embargo, la relación entre gobernantes y medios de comunicación no ha tenido siempre una óptima sintonía. Autores como Grebe (2007) y Sirvén (2012) sostienen que esta ilación entre ambos agentes es, en ocasiones, complicada porque los primeros no entienden la labor de los segundos cuya función y finalidad es informar a los ciudadanos de forma imparcial sobre las actuaciones gubernamentales. No obstante, la habitual práctica de los medios de comunicación dista en grandes ocasiones de alcanzar este loable fin. Para Casero-Ripollés (2012) la tarea 
periodística se basa frecuentemente en la exclusiva recogida de declaraciones y manifestaciones públicas de los políticos, lo que conlleva, entre otras consecuencias, la preferencia de fuentes procedentes de los propios políticos que, según Dader (2012), supone una imposición a los periodistas de la agenda temática gubernamental.

La aguda politización de los medios de comunicación en España resaltada por numerosas investigaciones se ha producido básicamente desde la consolidación de la democracia hasta la actualidad (López García, 2011; Sampedro y Seoane, 2008). Esta tendencia es perfilada desde dos frentes distintos, aunque no contrapuestos, sino vinculados uno con el otro. Por un lado, autores como Hanitizsch (2011) y Canel (2000) aseguran que esta politización no está relacionada directamente con los profesionales de los medios, sino con las empresas corporativas a los que pertenecen estos; mientras que, por otro lado, Casero-Ripollés (2008) considera que los medios de comunicación se identifican con una tendencia ideológica más que con un expreso y evidente apoyo a un determinado partido político.

El mecanismo de listas cerradas en los procesos electorales de regímenes parlamentarios conlleva, a priori, un mayor protagonismo de los partidos políticos que de los líderes; al contrario de lo que ocurre en los regímenes presidencialistas. A excepción de autores como Adam y Maier (2010), diversas investigaciones (Caprara, 2007; McAllister, 2007; Poguntke y Webb, 2005) consideran, no obstante, que en todo sistema democrático, independientemente del régimen establecido, se impone la personalización de los líderes. Un fenómeno motivado en gran parte por el funcionamiento de sus respectivos partidos que con gran frecuencia otorgan el protagonismo de sus acciones al líder, principalmente en el transcurso de las campañas electorales (Scarrow et al., 2000:122).

Otras investigaciones, finalmente, dirigen sus miradas hacia los medios de comunicación cuyo tratamiento informativo de los acontecimientos políticos provoca el impulso al papel principal de los líderes (Rico, 2009; Rahat y Sheafer, 2007) y más recientemente, se ha argumentado que son las redes sociales como Facebook y Twitter las que potencian esa personalización (Enly \& Skogerbø, 2013).

\section{OBJETIVOS}

Como respuesta corroborativa o de rechazo a la opinión emitida por la ciudadanía en las encuestas exploratorias ya mencionadas (El País, 2013; La Vanguardia, 2015), el objetivo que nos proponemos en este estudio es analizar el protagonismo mediático y comprobar la valoración que Ana Botella ha recibido en los principales periódicos madrileños de tirada nacional impresa: ABC, El Mundo, El País y La Razón durante cinco acontecimientos clave que han marcado su trayectoria política: además de su nombramiento y renuncia, el ya mencionado caso del Madrid Arena, el fracaso de Madrid como candidata a albergar los Juegos Olímpicos de 2020 y, finalmente, la larga huelga de los operarios del servicio de recogida de basuras. 
De este modo, se pretende comprobar si la valoración que ha hecho la prensa de la alcaldesa Ana Botella durante su mandato en Madrid ha sido positiva, negativa, neutra, o qué relevancia ha podido tener en las informaciones y opiniones expresadas por los medios en los momentos más importantes de su legislatura.

\section{METODOLOGÍA}

El análisis comparativo que aquí se presenta toma como base los cuatro periódicos madrileños de versión impresa y mayor tirada nacional: ABC, El Mundo, El País y la Razón. De ellos se seleccionaron aquellas piezas informativas y de opinión (artículos de columnistas, editoriales y viñetas) referidas a cinco acontecimientos que han marcado, en el plano mediático, la trayectoria política como alcaldesa de Ana Botella:

1. Su nombramiento: 26 de diciembre de 2011.

2. Suceso del Madrid Arena: noche del 1 al 2 de noviembre de 2012.

3. Descarte por el COI de la candidatura olímpica Madrid 2020: 7 de septiembre de 2013.

4. Huelga de basuras: Del 5 al 17 de noviembre de 2013.

5. Su renuncia oficial para la candidatura de elecciones municipales 2015: 9 de septiembre de 2014.

Para una acotación cronológica de estos hechos, se seleccionaron las piezas publicadas en los mencionados periódicos durante los intervalos siguientes:

1. Su nombramiento: Desde el 21 de noviembre de 2011 (fecha en que su antecesor, Alberto Ruiz-Gallardón se barajaba como ministro del nuevo gobierno salido de las urnas el 20 de noviembre) hasta el 31 de diciembre del mismo año.

2. Suceso del Madrid Arena: Desde el 2 de noviembre de 2012 hasta el 10 de enero de 2013 (dos días antes dimitió el vicealcalde Miguel Ángel Villanueva, mano derecha de Ana Botella).

3. Descarte de la candidatura olímpica Madrid 2020: Desde el 1 de septiembre (llegada de Ana Botella a Argentina, país donde se presentaron las candidaturas seleccionadas) hasta el 15 de septiembre (tras el fracaso y las reacciones).

4. Huelga de basuras: Del 1 al 30 de noviembre de 2013. Se ha seleccionado todo este mes para analizar también los prolegómenos y las secuelas que conllevó la protesta.

5. Renuncia: Desde el 10 de septiembre de 2014 (cuando se empiezan a hacer eco los periódicos) hasta el 15 de septiembre del mismo año.

En una segunda y última fase se ha realizado un análisis de todas las piezas recogidas. Los datos resultantes han sido codificados y registrados manualmente en dos bases de datos. Por una parte, se ha tomado como eje el formato de las piezas con una división entre los siguientes ítems:

1. Noticia: Con carácter meramente informativo. 
2. Opinión: Artículos realizados por un columnista o una firma especializada.

3. Editorial.

4. Viñeta: De carácter crítico-humorístico.

La segunda base de datos ha sido elaborada tras un análisis de contenido y, para ello, se clasificaron cada una de las piezas en alguna de estas variables:

1. Positivas: Con tendencia favorable a la figura política de Ana Botella.

2. Negativas: Con tendencia contraria a la figura política de Ana Botella.

3. Neutras: No se observa ningún tipo de trato de favor o de postura contraria a la figura de Ana Botella.

4. Piezas en las que Ana Botella no aparece, ni en los titulares ni en el texto.

\section{DISCUSIÓN Y RESULTADOS}

Entre los cinco acontecimientos escogidos, el suceso del Madrid Arena y la candidatura de Madrid 2020 han sido los más seguidos por la prensa con 674 y 429 piezas publicadas respectivamente. Sin embargo, si atendemos a los días en los que se han tratado los diferentes capítulos, es sin duda la candidatura olímpica la más observada, seguida del anuncio de la alcaldesa a no presentarse a las elecciones de 2014 (Gráf. 1).

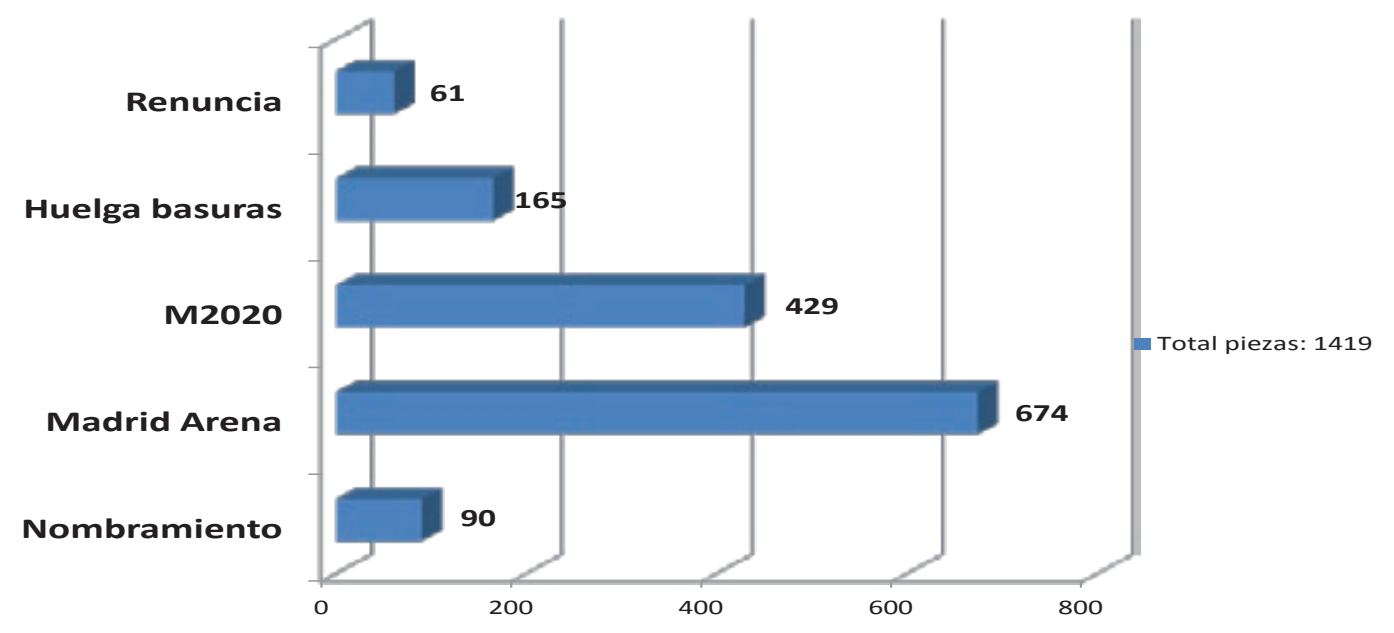

Gráfico 1: Total de piezas por acontecimiento

Fuente: Elaboración propia

La cobertura diacrónica que se ha seguido sobre el Madrid Arena en los diferentes periódicos elegidos ha sido la más amplia: del 2 de noviembre de 2012, día del trágico suceso, hasta el 10 de enero de 2013 tras la dimisión del vicealcalde Miguel Ángel Villanueva. Ello ha supuesto una media de 10 informaciones al día. Sin embargo, en el caso de la candidatura olímpica de Madrid para 2020 se ha producido 
una mayor densidad mediática ya que en la primera quincena de septiembre del 2013 se registró una media de 28,6 publicaciones diarias, resultado de un total de 429 piezas.

Por su parte, la renuncia se ha tratado entre los días 10 y 15 de septiembre de 2014 en 61 piezas, lo que significa 12,2 piezas al día, superando al caso del Madrid Arena. También se han estudiado durante un mes las publicaciones sobre la huelga de basuras del mes de noviembre de 2013, con un resultado de 165 piezas que suponen 5,5 al día, mientras que en el caso del nombramiento el 28 de diciembre de 2011, se han seguido los periódicos de un mes y una semana antes con un resultado de 90 publicaciones, lo que arroja un resultado de 2,4 diarias.

En todos los casos las piezas informativas superan siempre a los editoriales, las viñetas o las opiniones, donde se incluyen la carta de un lector que publicó El Mundo sobre el caso Madrid Arena y 6 columnas de opinión que escribió la propia Ana Botella en el diario ABC sobre la Candidatura Olímpica de Madrid (Gráf. 2).

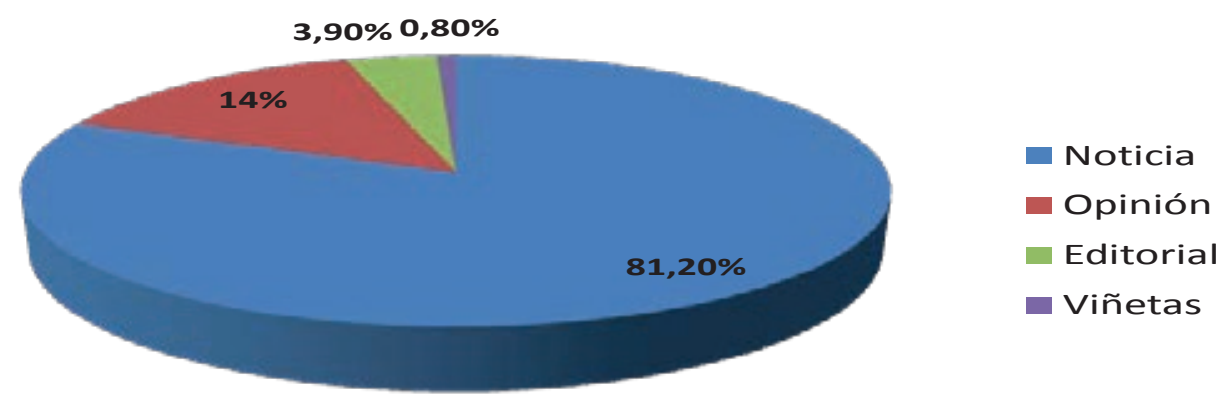

\section{Gráfico 2: Porcentajes totales de formatos \\ Fuente: Elaboración propia}

Los artículos de opinión son más numerosos también en los casos de Madrid 2020 y del Madrid Arena, con 96 y 60 respectivamente, mientras que en el caso del nombramiento de Ana Botella como alcaldesa han sido 26; 19 en la huelga de basuras, y 15 referidos a su renuncia a presentarse a las elecciones. De nuevo es el caso de Madrid 2020 el tema más recurrente con una media diaria de 6,4 piezas, mientras que en el resto de los casos es inferior a 1 por día.

Respecto a los editoriales, el caso del Madrid Arena ha reflejado 24, un 0,3 al día, seguido por la candidatura olímpica de Madrid, que en 15 días ha ofrecido 13 editoriales, un 0,8 al día; lo mismo que la renuncia de la alcaldesa con 4 editoriales en cinco días. Más testimonial ha sido el caso del nombramiento, con 2 editoriales, y la huelga de basuras con tan solo 1 . 
En estos cinco acontecimientos recogidos se observa también que se publican entrevistas en tres de ellos. Son 17 en el caso de Madrid 2020, 8 en el caso del Madrid Arena, y una en la huelga de basuras. Por su parte, las 12 viñetas se hacen presentes en tres acontecimientos: 6 sobre el Madrid Arena, 4 sobre la candidatura olímpica de Madrid y 2 por la renuncia de la alcaldesa (Gráf. 3).

\begin{tabular}{|l|r|r|r|r|r|}
\hline ACONTECIMIENTO & \multicolumn{1}{|l|}{ NOTICIAS } & OPINION & EDITORIAL & VIÑETA & TOTAL \\
\hline NOMBRAMIENTO & 62 & 26 & 2 & 0 & 90 \\
\hline M. ARENA & 600 & 43 & 24 & 6 & 673 \\
\hline M2020 & 316 & 96 & 13 & 4 & 429 \\
\hline BASURAS & 145 & 19 & 1 & 0 & 165 \\
\hline RENUNCIA & 40 & 15 & 4 & 2 & 61 \\
\hline
\end{tabular}

Gráfico 3: Cantidad de formatos por acontecimientos

Fuente: Elaboración propia

En cuanto a la valoración de las piezas publicadas, en términos generales destaca el amplio número de piezas $(61,4 \%)$ en las que no aparece el nombre de Ana Botella ni en el titular ni en el texto. En el 38,6\% restante, las piezas con una valoración neutra de la alcaldesa son sensiblemente las más frecuentes con un $18,9 \%$, mientras que las negativas integran un $11,2 \%$ y las positivas un $8,4 \%$.

En los casos más tratados, de las 429 piezas sobre Madrid 2020, en 304 (70,8\%) no aparece la alcaldesa, mientras que en el caso del Madrid Arena en 482 piezas de 674 (71,5\%), tampoco figura el nombre de Ana Botella. En la huelga de basuras el porcentaje es del $51,5 \%$ y que responde a 85 piezas de 165 totales; mientras que en el nombramiento lógicamente sí aparece la alcaldesa en todas ellas; igualmente, sobre su renuncia tan sólo en 1 de las 62 piezas no se cita a la alcaldesa.

Por otra parte, la mayoría de las piezas son neutras en los casos del nombramiento, Madrid 2020 y la huelga de basuras. Sin embargo predominan las valoraciones negativas en los acontecimientos referidos a su renuncia y al suceso de Madrid Arena (Gráf. 4). 


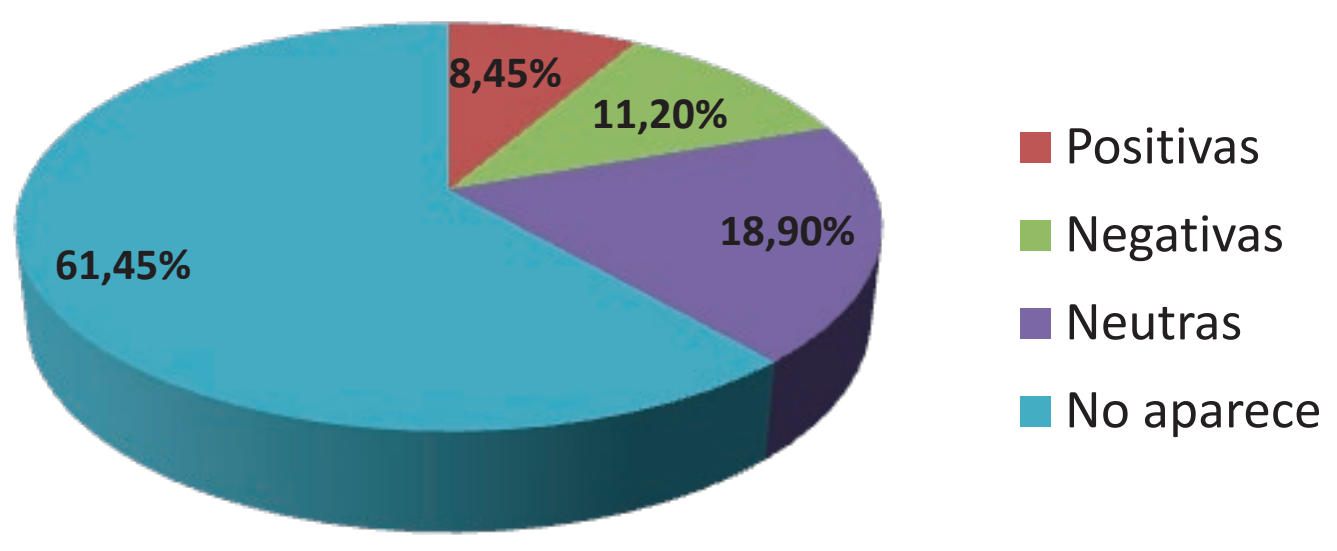

Gráfico 4: Valoración porcentual en todos los diarios

Fuente: Elaboración propia

Por periódicos, todos optan por difundir más piezas sobre Madrid 2020 y el Madrid Arena. $\mathrm{ABC}$ es el que más publica sobre este último suceso con 218 piezas, seguido por El Mundo con 181, y La Razón con 180, mientras que El País sorprende con prácticamente la mitad, 95 . En el lado contrario, todos dedican el menor número de piezas a la renuncia de Ana Botella, salvo El País que refleja todavía menos en el caso del nombramiento de la alcaldesa.

Es significativo que, en el tema del Madrid Arena, La Razón sea el periódico que más artículos de opinión pública, con 24, por delante de ABC, con 21, y por encima de $\mathrm{El}$ Mundo, con 14, y de El País, con solo 1. Sin embargo, es El Mundo el que sobresale con 14 editoriales, superando con creces a los 7 de ABC, los 2 de El País, y a uno solo de La Razón.

Según la valoración, lo primero que llama la atención es que La Razón no refleja piezas negativas en ningún caso, y las positivas superan al resto de parámetros, excepto en los casos de Madrid 2020 y Madrid Arena, donde las veces que no aparece el nombre de Ana Botella supera ampliamente al resto de valoraciones.

En el otro lado de la balanza, El País tan solo refleja una pieza positiva, y es precisamente en una opinión sobre el caso Madrid 2020 que contrasta con 6 negativas, 20 neutras y 41 donde no aparece el nombre de la alcaldesa. Destacan también en El País las 9 piezas negativas por una neutra de las 10 publicadas en el caso de la renuncia.

También El Mundo se muestra negativo en su mayoría de publicaciones. Tan solo 4 piezas son positivas mientras que las negativas superan a las positivas en los casos de la renuncia, el Madrid Arena y Madrid 2020. Eso sí, también destaca la amplia 
diferencia con las veces que no sale reflejada la alcaldesa en los casos de Madrid 2020, Madrid Arena y en la huelga de basuras.

Es el $A B C$ el más neutral y en todos los casos la valoración neutra supera al resto, aunque también se aprecia la mayoría de veces en los que no aparece la alcaldesa en los temas de Madrid 2020, Madrid Arena y la huelga de basuras (Gráf. 5).

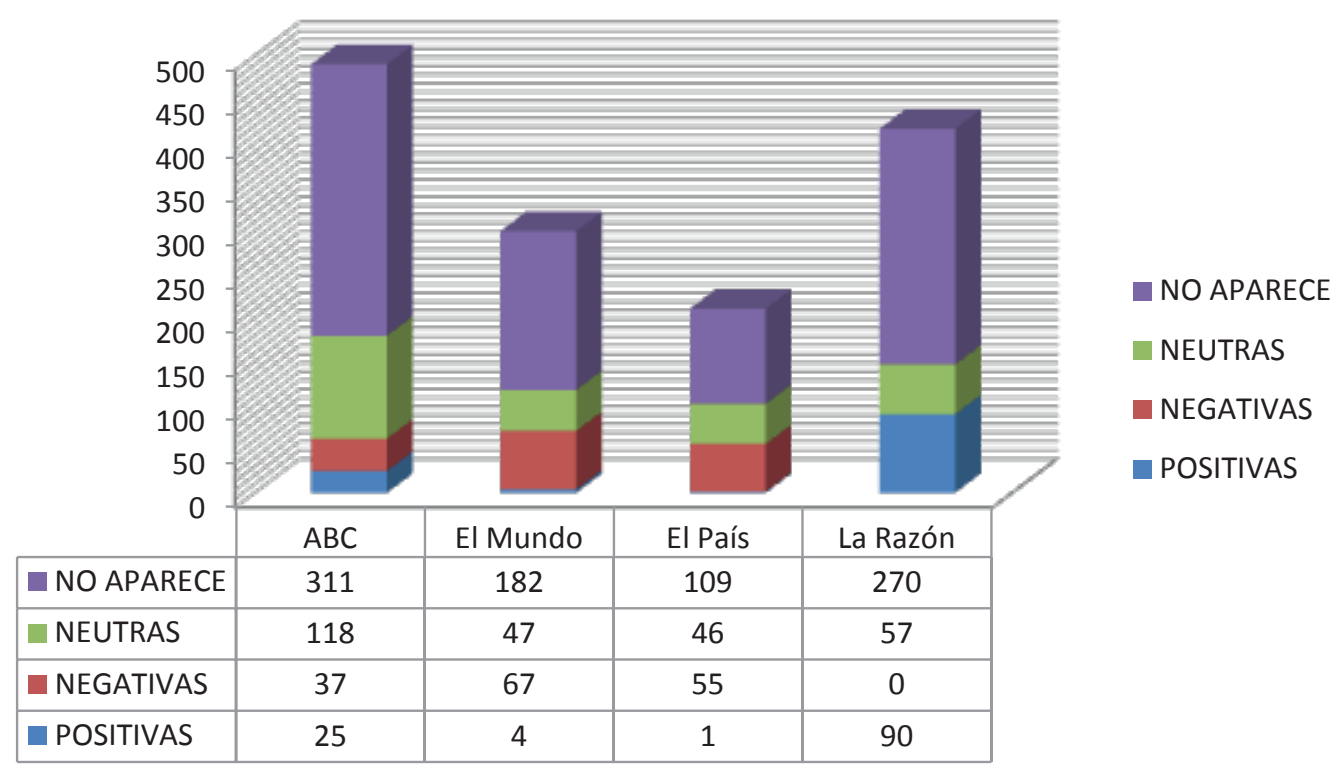

Gráfico 5: Valoración por diarios

Fuente: Elaboración propia

Según los casos planteados, tan solo en el nombramiento las piezas informativas que predominan son neutras, y las opiniones son positivas en su mayoría. Por el contrario en el tema de la renuncia de Ana Botella, aunque las piezas informativas que predominan son neutras, las opiniones son en su mayoría negativas (Gráf. 6). 


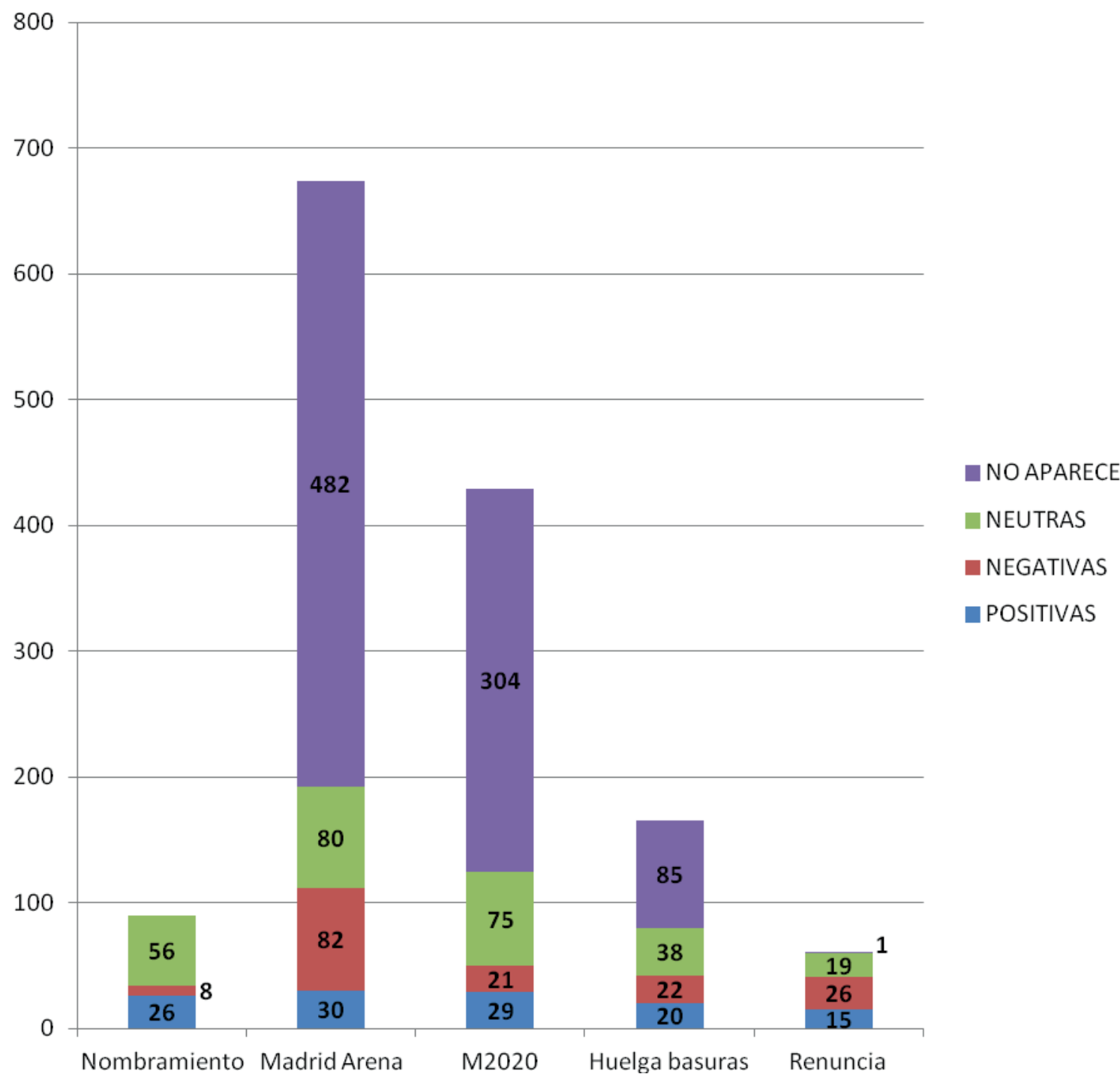

Gráfico 6: Valoración por acontecimientos

Fuente: Elaboración propia

En los temas de la huelga de basuras, las piezas informativas donde no sale reflejada la alcaldesa son claramente superiores, pero en el caso de las opiniones hay un número similar de valoraciones positivas y negativas. En cuanto a los temas de Madrid 2020 y el Madrid Arena, las piezas, tanto informativas como de opinión, donde no sale reflejada la alcaldesa superan ampliamente al resto de valoraciones.

\section{CONCLUSIONES}

Si bien se observa un notable grado de imparcialidad en los periódicos estudiados, la figura de Ana Botella se enfoca, en general, desde un punto vista crítico más negativo que positivo, hecho que coincide con los resultados de las encuestas explorativas realizadas entre la opinión pública por determinados diarios. Esta 
perspectiva es variable en función de la línea editorial marcada en cada publicación: La Razón es el periódico más clemente mientras que El País es el que muestra la postura más inflexible hacia las acciones de la alcaldesa. Esta diferencia de criterio entre unos periódicos y otros es, en cierto modo, previsible debido, precisamente, a la diversidad en la tendencia ideológico-política de los diarios estudiados.

Lo que sí resulta más llamativo es el escaso protagonismo que ha tenido Botella en los principales acontecimientos que han marcado su gestión, a pesar de ser la principal cabeza visible de la administración municipal de la capital. Esta relevancia ha quedado relegada por otros actuantes: miembros de su gobierno, el ayuntamiento como institución y distintas figuras mediáticas que, en un grado mayor en algunos casos, pero sobre todo igual o menor que la propia alcaldesa, también estaban involucradas y/o eran responsables de los asuntos investigados.

De los cinco temas elegidos para este estudio, los trágicos sucesos del Madrid Arena y el descarte de Madrid como sede olímpica para 2020 han sido los más seguidos por la prensa seleccionada, lo que refleja la importancia de estos acontecimientos en la legislatura de Ana Botella. Por otra parte, si atendemos a los datos de la proporción de piezas publicadas en relación a los días de seguimiento, es el caso de Madrid 2020 el más tratado, seguido por la huelga de basuras en la capital.

El hecho de que en una amplia mayoría las piezas, 872 de las 1.419 en total $(61,45 \%)$ no hagan una referencia explícita a la alcaldesa de Madrid, sin reflejar su nombre ni en el titular ni en el texto, confirma una escasa presencia de Ana Botella en la prensa, incluso en los acontecimientos más importantes de su mandato.

Sin contar las piezas que no reflejan el nombre de Ana Botella, la mayoría tienen una valoración neutra, 268, un 18,8\%; mientras que las 159 negativas suponen un $11,2 \%$, y las positivas son 119 , un $8,3 \%$.

Por periódicos, es La Razón el diario que mejor valoración hace de la alcaldesa con 90 piezas positivas, un $21,5 \%$ frente a ninguna negativa, mientras que es El País quien ofrece una valoración más negativa con 55 piezas con este criterio, un 26,9\% en contraste con solo una positiva $(0,4 \%)$.

Los casos del nombramiento y de la renuncia cuentan con más porcentaje de piezas positivas. El caso del nombramiento refleja 26 piezas positivas $(28,8 \%)$, seguido de la renuncia con 15 , un $24,1 \%$, de la huelga de basuras, con 20 , un $12,1 \%$, mientras que Madrid 2020 refleja un 6,7\% de piezas positivas con 29, y el Madrid Arena un $4,4 \%$ con 30 .

La Razón y $A B C$ son los dos diarios que más piezas de opinión han difundido, otorgando más espacio al análisis que El Mundo y El País. La Razón ha publicado 98 piezas de opinión, un 23,5\%, frente a las 98 de ABC: un 19,9\%; las 48 de El Mundo, lo que supone un $16 \%$; y las 17 de El País, un $8 \%$, siendo una vez más significativo 
el caso de la candidatura olímpica para 2020, con 42 opiniones en La Razón (28,3\%) y 41 en $A B C(25,1 \%)$.

\section{REFERENCIAS}

Adam, S. \& Maier, M. (2010). Personalization of politics: A critical review and agenda for research.

Aira, A. (2009). Los spin doctors: Cómo mueven los hilos los asesores de los líderes políticos. Editorial UOC.

Canel, M. J.; Rodríguez Andrés, R. y Sánchez Aranda, J. J. (2000): Periodistas al descubierto. Retrato de los profesionales de la información. Madrid: CIS.

Caprara, G. V. (2007). The personalization of modern politics. European Review, 15(02), 151-164.

Casero Ripollés, A. (2012). El periodismo político en España: algunas características definitorias.

Recuperado

de

http://repositori.uji.es/xmlui/bitstream/handle/10234/80553/Casero 19 46.pdf?sequence $=1$

Casero-Ripollés, A. (2008): "Modelos de relación entre periodistas y políticos: la perspectiva de la negociación constänte Estudios sobre el mensaje periodístico, 14: 111- 128.

Castillo, J. (2003). Hugo Chávez y los medios de comunicación: la guerra" hiperreal" venezolana. Historia y comunicación social, (8), 21-34.

Dader, J. L. (2012): "Periodismo político y política del periodismo: imaginando un futuro digno y sostenible". En Berrocal Gonzalo, S. y Campos Domínguez, E. (eds): La investigación en periodismo político en el entorno de los nuevos medios. Madrid: SEP.

Domínguez, T. (2012). La imagen de Vladimir Putin en la prensa rusa. Zer-Revista de Estudios de Comunicación, 6(10).

El País. (2013, 14 de mayo) Intención de voto y valoración de líderes en Madrid. Recuperado de http://elpais.com/elpais/2013/05/14/media/1368548475 962412.html.

Enli, G. S. \& Skogerbø, E. (2013). Personalized campaigns in party-centred politics: twitter and Facebook as arenas for political communication. Information, Communication \& Society, 16(5), 757-774.

Grebe, R. (2007). Evo Morales y los medios. Chasqui. Revista Latinoamericana de 
Comunicación, (98), 10-15.

Gunther, R. \& Mughan, A. (Eds.). (2000). Democracy and the media: a comparative perspective. Cambridge University Press.

Hanitzsch, T. (2011): Populist disseminators, detached watchdogs, critical change agents andopportunist facilitators: Professional milieus, the journalistic field and autonomy in 18 countries. International Communication Gazette, 73 (6): 477-494. DOI: $10.1177 / 1748048511412279$

Horton, D. \& Richard Wohl, R. (1956). Mass communication and para-social interaction: Observations on intimacy at a distance. Psychiatry, 19(3), 215-229. Recuperado de http://www.participations.org/volume\%203/issue\%201/3 01 hortonwohl.htm

La Vanguardia (2015, 11 de febrero). Encuesta sobre la valoración de Ana Botella al frente de la alcaldía. Recuperado de http://www.lavanguardia.com/participacion/encuestas/20140910/54415827445/comovaloras-ana-botella-alcaldesa-ayuntamiento-madrid.html.

López García, (2011). Política binaria y SPAM electoral: elecciones generales 2008: nuevas herramientas, viejas actitudes. Tirant lo Blanch, 2011.

McAllister, I. (2007). The personalization of politics. The Oxford handbook of political behavior, 571-588. Recuperado de http://politicsir.cass.anu.edu.au/staff/mcallister/pubs/personal.pdf

Neveu, E. (2004): Sociologie du journalisme. Paris: La Découverte.

Poguntke, T. \& Webb, P. (2005). The presidentialization of politics in democratic societies: a framework for analysis. The presidentialization of politics: a comparative study of modern democracies, 1-25. Recuperado de http://www.logobook.ru/af/11028194/2537/0199252017 sample.pdf

Rahat, G. \& Sheafer, T. (2007). The personalization (s) of politics: Israel, 1949-2003. Political Communication, 24(1), 65-80. Recuperado de http://eshkol.huji.ac.il/doc/Gideon\%20RahatTamir\%20SheaferThe\%20Personalization\%20of\%20Politics\%20Israel\%201949-2003.pdf

Rico, Guillém. (2009). Líderes políticos, opinión pública y comportamiento electoral en España (No. 270). CIS.

Sampedro, V. y Seoane, F. (2008): "The 2008 Spanish general elections: "Antagonistic Bipolarization" geared by presidential debates, partisanship, and media interests". International Journal of Press/Politics, 13(3): 336-344. DOI: 
Scarrow, S. E., Webb, P. \& Farrell, D. M. (2000). From social integration to electoral contestation: The changing distribution of power within political parties. Parties without Partisans: Political Change in Advanced Industrial Democracies, 129-153.

Sirvén, P. (2012). Perón y los medios de comunicación: La conflictiva relación de los gobiernos justicialistas con la prensa 1943-2011. Sudamericana.

Valdez, A. \& Rivera, R. (2009). Obama, en la Prensa Latinoamericana. Revista Latina de comunicación social, (64), $10 . \quad$ Recuperado de http://www.revistalatinacs.org/09/art/10 80921 Guadalajara/Valdez Zepeda y Rivera.h $\underline{\mathrm{tml}}$

Van Dalen, A. (2012): The people behind the political headlines: A comparison of political journalists in Denmark, Germany, the United Kingdom and Spain. International Communication Gazette, 74 (5): 464-483. DOI: $10.1177 / 1748048512445154$

\section{AUTORES:}

\section{Beatriz Catalina-García}

Doctora en Ciencias de la Comunicación por la Universidad Rey Juan Carlos (2011) y Licenciada en Ciencias Políticas por la UNED (2014). Profesora Visitante del Área de Periodismo de la URJC. Ha participado en el Proyecto Nacional de I+D+I "Análisis de uso y consumo de medios y redes sociales en Internet entre los adolescentes españoles. Características y prácticas de riesgo". Ha trabajado como periodista en diferentes medios de comunicación. Algunos de sus artículos científicos publicados recientemente: "Los riesgos de los adolescentes en Internet: los menores como actores y víctimas de los peligros de Internet" (2014): "Hábitos de uso en Internet y en las redes sociales de los adolescentes españoles (2013); "Herramientas interactivas y participación ciudadana en los Ayuntamientos españoles" (2013). [ORCID] [GS].

\section{José Gabriel Fernández-Fernández}

Doctor en Ciencias de la Información por la Universidad Complutense, Master de Periodismo Económico por la Complutense. Profesor en la Universidad Rey Juan Carlos. Licenciado en Ciencias de la Información por la Universidad de Navarra y autor de varias publicaciones sobre periodismo especializado en economía y en deporte, ha participado en diferentes congresos internacionales sobre estas especialidades. Ha trabajado como redactor en la sección local de Diario 16 entre 1995 y 1998 y como redactor en la sección de economía del canal 24 Horas de TVE entre los años 1998 y 2005. Es colaborador habitual en madridiario.es y en Radio Marca, y ha colaborado en Radio Intereconomía así como en diferentes revistas especializadas sobre deporte y sobre economía. 